\title{
Amplification of a Laser Beam for the Fast Ignition of a Thermonuclear Target
}

\author{
Friedwardt Winterberg \\ University of Nevada, Reno, Nevada, 1664 N. Virginia St. MS 220, Reno, NV 89523, USA \\ Reprint requests to Prof. F. W.; Fax: (775) 784-1398; E-mail winterbe@physics.unr.edu \\ Z. Naturforsch. 62a, $601-604$ (2007); received May 30, 2007
}

\begin{abstract}
It is proposed to simultaneously compress a thermonuclear target and amplify a laser beam by a single $z$-pinch discharge. The laser beam is imploded and amplified by a cylindrical convergent shock wave inside a capillary, transforming it into a soft X-ray pulse for the fast ignition of the thermonuclear target. The target is compressed inside a liner by the z-pinch current. The capillary is attached to one end of the cylindrical target, and is protected by a radial wire spoke array fast opening switch against its premature implosion by the convergent shock wave. The $z$-pinch can be stabilized by placing it into a powerful vortex.
\end{abstract}

Key words: Fast Ignition; z-Pinch; Laser Amplification.

\section{Introduction}

The approach taken by most research groups towards the realization of the fast ignition of a small DT (deuterium-tritium) target is by ablative compression to $\sim 10^{3}$ times solid density, followed by its hot spot ignition with $\mathrm{a} \sim 100 \mathrm{~kJ}$ petawatt laser $[1,2]$. The other, less vigorously pursued approach is by magnetic compression inside a linear pinch discharge, followed by the hot spot ignition at one end of the pinch discharge with a petawatt laser [3]. In both cases, a thermonuclear detonation wave is launched from the hot spot, burning up the remaining part of the DT target. The first case requires that for the hot spot $\rho r \geq 1 \mathrm{~g} / \mathrm{cm}^{2}$, where $\rho$ is the density of the compressed DT target and $r$ the radius of the hot spot. In the second case the target density can be less, but the pinch current must there be of the order $10^{7} \mathrm{~A}$. In either case, these conditions are necessary to keep the charged fusion products entrapped inside the burning plasma. Because in the second case the target has the shape of a long cylinder, very large gains seem to be possible in principle, provided the DT pinch column can be kept stable for the short time the detonation wave passes through it with a velocity approximately $1 / 10$ the velocity of light.

To keep the thermonuclear microexplosion at a safe distance from the wall of the cavity confining the microexplosion, is for obvious reasons no problem in the first case. But it is a problem in the second case, be- cause one needs a transmission line from the wall to the electrodes of the pinch discharge, which, through its absorption of energetic neutrons from the DT thermonuclear reaction, is transformed into a hot plasma, leading to a blast wave. This problem though can be solved by the concept of the inverse diode invented by I. Smith [4].

Replacing the very expensive $100 \mathrm{~kJ}$ petawatt laser by something less expensive would be highly desirable. In one idea proposed by Murakami and Nagatomo called impact ignition $[5,6]$, a small solid projectile is ablatively accelerated by a 100 terawatt laser to a velocity of $\sim 10^{3} \mathrm{~km} / \mathrm{s}$, which upon its impact on the highly compressed DT target generates a hot spot, launching from it a thermonuclear detonation wave. As a more efficient alternative to this rocket-like acceleration I had proposed a gun-like acceleration making use of the magnetized booster target concept [7]. The general advantage of impact fusion, and this also applies to impact ignition, is that it permits the kinetic energy of a projectile to increase relatively slowly.

One might think that an intense ion beam, generated by the laser bombardment of a thin foil, might be a better alternative to impact ignition. But this is not so because of the poor laser-to-kinetic ion beam energy transfer efficiency, which is, at best, a few percent. A totally different situation arises at very high laser intensities, where in a "laser piston" regime the intense laser light pushes forward (by its radiation pressure) a block of condensed matter [8-10]. Here the laser-to- 
kinetic energy transfer efficiency is high, but requires the very high laser light intensities that the impact ignition scheme tries to avoid.

In this communication I propose the amplification of a $\sim 10 \mathrm{~kJ}-100$ terawatt laser pulse into a $100 \mathrm{~kJ}$ petawatt soft X-ray pulse by the compression of the laser pulse inside an imploding capillary for the fast ignition of a DT target by a single high current pinch discharge.

\section{Description of the Novel Fast Ignition Concept}

As shown in Fig. 1, a long DT cylinder with an initial radius $R_{0}$ is attached at its end to a metallic cylinder, with a capillary of an initial radius $r_{0}$ at its core.

A large current, drawn from a Marx generator and coming from the right, flows over the DT cylinder compressing it from its initial radius $R=R_{0}$ to its final radius $R=R_{1}$. In reaching the end of the DT cylinder on the left, the current flows in the radial direction over a spoke-like array of wires, in a configuration proposed by the author in a previous publication [11]. The implosion velocity of the DT cylinder is by order of magnitude equal to the Alfvén velocity $v_{\mathrm{A}}=B \sqrt{4 \pi \rho}$, where $B$ is the magnetic field strength and $\rho$ the density. For a current of $10^{7} \mathrm{~A}, R_{0}=5 \times 10^{-2} \mathrm{~cm}$, and a density of $\rho \sim 1 \mathrm{~g} / \mathrm{cm}^{3}$, one has by order $v_{\mathrm{A}} \sim 10^{8} \mathrm{~cm} / \mathrm{s}$, with the
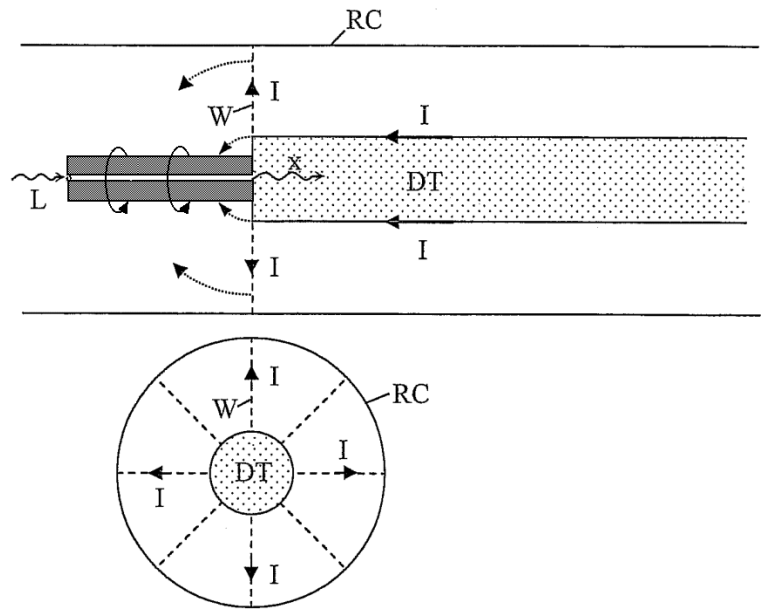

Fig. 1. Amplification of a laser beam by a convergent cylindrical shock wave for the fast ignition of a dense z-pinch. I, current compressing the DT cylinder; RC, return current conductor; $\mathrm{W}$, thin exploding wires forming an opening switch to let the current I pass over the outer surface of the cylinder containing the capillary $\mathrm{C}$; $\mathrm{L}$, laser beam shot into the capillary compressed by a convergent cylindrical shockwave. implosion time $\tau_{\mathrm{imp}} \sim R_{0} / v_{\mathrm{A}} \sim 5 \times 10^{-9} \mathrm{~s}$. In flowing over the radial wire array, the magnetic pressure created by the large current accelerates the array to the left, thereafter imploding it onto the cylinder containing the capillary. As in the dense plasma focus, the wire array acts as a fast switch, directing the current to flow over the cylinder containing the capillary, launching from its surface towards its center a convergent cylindrical shockwave. For a convergent cylindrical shockwave, the pressure rises in proportion to $r^{-0.4}$, where $r$ is the distance from the center of convergence [12]. The time the shockwave implodes the capillary can be much shorter than the time the convergent shockwave needs to reach the capillary.

A laser beam coming from the left and entering the capillary of length $l$ is compressed in the time $\tau_{l} \sim l / c$, which must be about equal to the implosion time $\tau_{\text {imp }} \sim r_{0} / v_{\mathrm{A}}$ of the capillary. One thus obtains the important relation

$$
l / r_{0} \sim c / v_{\mathrm{A}} .
$$

Setting $r_{0} \sim 10^{-2} \mathrm{~cm}, v_{\mathrm{A}} \sim 10^{8} \mathrm{~cm} / \mathrm{s}$, one finds that $l \sim$ $3 \mathrm{~cm}$.

Following its implosive compression and amplification, the laser beam pulse is transformed into an incoherent soft X-ray pulse, which is shot into the left end of the compressed DT cylinder, creating a hot spot for its fast ignition.

\section{Imploding Capillary as Dynamic Hohlraum}

In indirect hohlraum configurations, one or more laser beams dissipate their energy inside a cavity, transforming the non-absorbed laser light into soft X-rays. In dynamic hohlraum configurations the soft $\mathrm{X}$-ray containing hohlraum is subsequently imploded, amplifying its energy. The absorption and re-emission of the laser light from the inner wall of the hohlraum is an irreversible process, with an efficiency $\eta<1$. Experiments with hohlraums show that by order of magnitude $\eta \approx 0.1$.

If the incoming laser light has an energy $W_{0}$, the energy converted into soft $\mathrm{X}$-rays is $\eta W_{0}$, with the hohlraum radiation temperature $T_{\mathrm{h}}$ given by the StefanBoltzmann law for black body radiation:

$$
a T_{\mathrm{h}}^{4}=\eta W_{0}, \quad a=7.67 \times 10^{-15} \mathrm{erg} / \mathrm{cm}^{3} \mathrm{~K}^{4},
$$

and the average frequency $\bar{v}$ of this radiation given by Wien's law ( $k$, Boltzmann constant):

$$
h \bar{v}=k T_{\mathrm{h}} .
$$


The hohlraum here is a capillary of initial radius $r_{0}$ and length $l$, with the capillary imploded from its initial radius $r_{0}$ to a smaller radius $r<r_{0}$. If the implosion is fast enough, i.e. the time for the implosion shorter than the time the radiation is lost by absorption in the wall of the capillary, the radiation behaves more like a gray body radiation where the photon mean free path is large compared to $l$. If $n$ is the number of atoms vaporized from the wall of the capillary and entering the capillary, and if $\sigma_{\mathrm{p}} \approx 10^{-18} \mathrm{~cm}^{2}\left(\sigma_{\mathrm{p}} \sim \alpha r_{\mathrm{B}}^{2}, \alpha=1 / 137\right.$, $r_{\mathrm{B}} \approx 10^{-8} \mathrm{~cm}$, Bohr radius) is the absorption cross section of the photons, one has for the photon mean free path $\lambda_{\mathrm{p}}=1 / n \sigma_{\mathrm{p}} \sim\left(10^{18} / n\right) \mathrm{cm}$. To have gray body radiation requires that $n<\left(10^{18} / l\right) \mathrm{cm}^{-3}$. For a $10^{-10} \mathrm{~s}$ laser pulse with $l \approx 3 \mathrm{~cm}$, one has $n<3 \times 10^{17} \mathrm{~cm}^{-3}$.

During the implosion of the capillary, the energy of the soft X-ray photon gas rises in proportion to $r^{-4 / 3}$, as for a monatomic gas where $\gamma=5 / 3$, and one has

$$
\frac{W_{x}(r)}{W_{x}\left(r_{0}\right)}=\left(\frac{r_{0}}{r}\right)^{4 / 3} \text {. }
$$

For an efficiency $\eta<1$, the laser energy $W(r)$ and power $P(r)$ are

$$
\frac{W_{x}(r)}{W_{0}\left(r_{0}\right)}=\frac{P(r)}{P_{0}}=\eta\left(\frac{r_{0}}{r}\right)^{4 / 3},
$$

where $W_{0}$ and $P_{0}$ are the initial laser energy and power, respectively. For the example $\eta=0.1, r_{0} / r=30$, one finds that $W(r) / W_{0}=P(r) / P_{0} \approx 10$, that is a tenfold amplification. If the soft X-ray pulse released from the capillary following its implosive compression lasts $10^{-10} \mathrm{~s}$, and if this energy is $W_{x}(r) \approx 10^{5} \mathrm{~J}$, as it is required for the fast ignition of the DT cylinder, one would need $W_{0} \sim 10^{4} \mathrm{~J}$ for the incoming laser energy of photons in the optical regions, amplified tenfold into soft X-ray photons. If the laser energy is supplied in $\sim 10^{-10} \mathrm{~s}$, the laser power would be $10^{14} \mathrm{~W}$, amplified into a $10^{15} \mathrm{~W}$ soft X-ray pulse.

The energy of the $10^{5} \mathrm{~J}=10^{12} \mathrm{erg}$ soft X-ray pulse must be of the same order of magnitude as the magnetic energy in the capillary. With $B=0.2 I / r$ this energy is

$$
E=\pi r^{2} l B^{2} / 8 \pi=(1 / 2) 10^{-2} I^{2} l .
$$

For $I=10^{7} \mathrm{~A}$ and $l=3 \mathrm{~cm}$ it is of the order $10^{12} \mathrm{erg}=$ $10^{5} \mathrm{~J}$. However, because the efficiency is only $10 \%$ $(\eta=0.1)$, ten times more energy is needed.

The thermonuclear yield depends only on the length of the $z$-pinch channel along which a thermonuclear
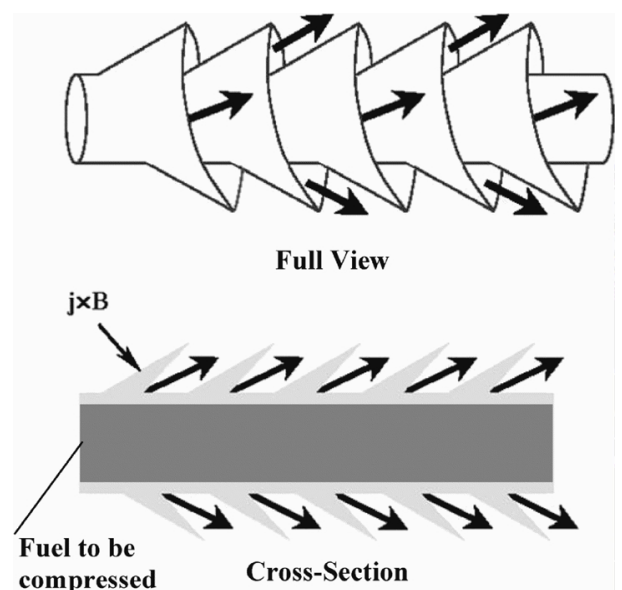

Fig. 2. A corrugated helical sawtooth-shaped liner with a solid DT core, to stabilize against the $m=0$ and $m=1 \mathrm{mag}$ netohydrodynamic instabilities and against the RayleighTaylor instability. Jets forming from the corrugated surface create shear and rotational flow.

detonation wave propagates, once it is ignited by the soft X-ray pulse at its one end.

\section{Stability}

The feasibility of the proposed concept largely depends on its stability. For the pinch discharge channel it is the $m=0$ (sausage) and $m=1$ (kink) instability, in addition to the Rayleigh-Taylor instability. All these three instabilities can be overcome by placing the pinch in the center of a strong potential vortex.

The $m=1$ pinch instability comes from Maxwell's equation curl $\mathbf{B}=0$ outside the pinch discharge channel, with the magnetic field, and with it the magnetic pressure, increasing with a decreasing distance from the center of curvature of the magnetic field lines. In a potential vortex where curlv $=0$, the same is true with regard to the velocity, except that an increase in the velocity leads by Bernoulli's theorem to a reduction in the pressure. Therefore, the pinch discharge channel is stabilized against the $m=1$ kink instability if $v>v_{\mathrm{A}}$.

Similar, because of $\oint \mathbf{B} \cdot \mathrm{d} \mathbf{r}=$ const. (constancy of current), and because of $\oint \mathbf{v} \cdot \mathrm{d} \mathbf{r}=$ const. (constancy of circulation), the pinch is stabilized as before against the $m=0$ sausage and Rayleigh-Taylor instability, if $v>v_{\mathrm{A}}$. These qualitative conclusions have been confirmed by Wanex et al. [13] in a detailed numerical analysis.

What remains is the question of how to generate such a powerful vortex where $v>v_{\mathrm{A}}$. One way how 
this can be done was proposed by the author [14], by placing the pinch inside a corrugated helical liner as shown in Figure 2. There, jetting from the corrugated surface by resistive ablation during its compression leads to its rapid rotation and axial shear flow. Because $v_{\mathrm{A}}$ is smaller in the dense metallic liner than in the lower density plasma, smaller rotation ve-

[1] N. G. Basov, S. Yu. Guskov, and L. P. Feoktistov, J. Sov. Laser Res. 13, 396 (1992).

[2] M. Tabak, J. Hammer, M.E. Glinsky, W.L. Kruer, S.C. Wilks, J. Woodworth, E. M. Campbell, D. Perry, and R. J. Mason, Phys. Plasmas I, 1626 (1994).

[3] F. Winterberg, Z. Naturforsch. 53a, 933 (1998).

[4] I. Smith, in: Proceedings of the International Topical Conference on Electron Beam Research and Technology (Ed. G. Yonas), 3-6 November 1975, Albuquerque, New Mexico, SAND76-5122, Vol. I, pp. $472-489$.

[5] M. Murakami and H. Nagatomo, Nucl. Instr. Methods Phys. Res. A 544, 67 (2005).

[6] M. Murakami, H. Nagatomo, T. Sakaiya, H. Azechi, S. Fujioka, H. Shiraga, M. Nakai, K. Shigemori, H. Saito, S. Obenschain, M. Karasik, J. Gardner, J. Bates, D. Colombant, J. Weaver, and Y. Aglit- locities are required than the Alfvén velocity in the plasma.

\section{Acknowledgement}

This work has been supported in part by the U.S. Department of Energy under Grant No. DE-FG0206ER54900. skiy, Plasma Phys. Controlled Fusion 47, B815 (2005).

[7] F. Winterberg, Phys. Plasmas 13, 112702 (2006).

[8] H. Hora, J. Badziak, F. P. Boody, R. Höpfl, K. Jungwirth, B. Králikova, J. Krása, L. Láska, P. Parys, V. Perina, M. Pfeifer, K. Rohlena, J. Skála, J. Ullschmied, J. Wolowski, and E. Woryna, Opt. Commun. 207, 333 (2002).

[9] T. Esirkepov, M. Borghesi, S. V. Bulanov, G. Mourou, and T. Tajima, Phys. Rev. Lett. 92, 175003-1 (2004).

[10] J. Badziak, S. Glowacz, S. Jablonski, P. Parys, J. Wolowsi, and H. Hora, Laser and Particle Beams 23, 401 (2005).

[11] F. Winterberg, Z. Naturforsch. 55a, 909 (2000).

[12] G. Guderley, Luftfahrtforschung 19, 302 (1942).

[13] L.F. Wanex, V. I. Sotnikov, and J. N. Leboeuf, Phys. Plasmas 12, 042101 (2005).

[14] F. Winterberg, Z. Naturforsch. 54a, 459 (1999). 